\title{
A Method for Prediction of Structure-borne Noise Radiated from Concrete Railway Viaducts
}

\author{
Masanori HANSAKA, Ph.D. \\ Daigo SATO \\ Senior Researcher, Laboratory Head, Researcher, \\ Shogo MAMADA \\ Vibration Isolating Material Laboratory, Materials Technology Division \\ Minoru SUZUKI \\ Assistant Senior Researcher, \\ Vibration Isolating Material Laboratory, Materials Technology Division \\ Kiyoshi NAGAKURA, Ph.D. \\ Senior Researcher, \\ Laboratory Head, Sound Analysis Laboratory, Environmental Technology Division

\begin{abstract}
Prediction of structure-borne noise has been a significant issue for railway viaducts, since it causes an excessive degree of noise attributable to structural member vibration. A new method of flexural vibration analysis expanded to cover whole structure for the prediction of structure-borne noise has therefore been developed. After calculation and measurement of noise in the vicinity of a floor slab of a concrete viaduct constructed on a Shinkansen line, it was revealed that the proposed method can be used as a practical means for predicting structure-borne noise.
\end{abstract}

Keywords: concrete viaduct, structure-borne noise, prediction of noise, analysis of vibration

\section{Introduction}

Railway structures such as concrete viaducts and steel bridges cause an excessive degree of noise, since in addition to rolling and aerodynamic noises generated anywhere in railway line, structure-borne noise is also generated by the vibration of those structures' members. Structure-borne noise is one of the main subjects to be settled out because it occasionally makes a high contribution to the trackside noise directly transmitted around the trackside while other noises such as rolling and aerodynamic noises are prevented ordinarily by the noise barrier. In order to develop effective countermeasures, it is necessary to understand the mechanism causing the physical phenomenon occurring around the structures, in order to develop a method to predict the outcomes of the phenomenon. Considering that structure-borne noise is generated by several members of the structure vibrating, first, a vibration analysis has to be made for the structure in question. After evaluation, it has been determined that the current vibration analysis methods, such as FEM (Finite Element Method) are unsuitable in their current technical level for the prediction of structure-borne noise for such reason that it requires to analyze vibrations of structure accurately up to $\mathrm{kHz}$ range, however, the $\mathrm{kHz}$ range is so broad that the current methods can't guarantee accurate solutions for very large structures.

This study therefore used the flexural vibration analysis, which was a confirmed calculation method applicable to individual members of a structure to analyze the vibration accurately up to $\mathrm{kHz}$ range. Using this method as a base, we modified it so as to be suitable for the prediction of structure-borne noise by extending it to a method which could analyze the vibration of whole structures [1].

On comparing predicted values, obtained with this new method for vibration analysis on a Shinkansen line concrete viaduct, with actual values from the floor slab center-point, the accuracy of the new method proved to be acceptable. The main reasons suggested for why the developed method can calculate structural vibration in the high frequency range are as follows: firstly, the developed method breaks the structure down into members represented by simple models, reducing the degrees of freedom in an equation of motion which increases calculation efficiency. Secondly, because the method introduces a trial function which takes into account the vibration mode of each member, which means that numeric matrix with the equation of motion of the structure in question can be obtained by treating each vibration mode as an element rather than dividing the spatial area into each small element.

A method for predicting structure-borne noise was then developed on the basis of the vibration velocity solutions obtained from this extended flexural vibration analysis. This paper describes the developed structure vibration analysis, sketches the outline of the subsequently designed structure-borne noise prediction method and reports on the results of verification by comparing the calculated and measured values of vibration and noise of a concrete viaduct constructed on a Shinkansen line. 


\section{Conventional methods for analyzing structure vibrations}

The main methods for analyzing structure vibrations used to date include the Finite Element Method (FEM) and the Statistical Energy Analysis (SEA). Both these methods were evaluated in order to determine their applicability to a method for predicting structure-borne noise. The results of these evaluations revealed the following major drawbacks:

(1) Finite Element Method (FEM) [2]

After dividing the subject for analysis into small elements, the Finite Element Method (FEM) involves producing an approximate dynamic equilibrium equation for each element. The total equation obtained from synthesizing these individual element equations is then used to find a solution in relation to the whole subject. Predicting structure-borne noise requires vibration analysis per $\mathrm{kHz}$ frequency range, however, in order to analyze the vibration accurately by FEM in such high frequency region, the subject should be divided into infinitely fine elements (elements are usually $1 / 6$ of the size of a wave length)*. Consequently the number of elements produced and related equations would be huge. For the calculation of vibration of structure by FEM in the range of $\mathrm{kHz}$, not only would the calculation time increase to an unmanageable level, but also it would be practically impossible to obtain the smallness in size of each element required for calculation accuracy due to the limited capacity of the computer, which causes a decrease of accuracy of FEM calculation in high frequency region. Moreover, the vast increase in number of elements causes errors such as cancellation of significant digits**. For these reasons, given current technical constraints, FEM was deemed unsuitable for accurate structure vibration analysis in a high $\mathrm{kHz}$ frequency range.

(2) Statistical energy analysis (SEA) [3]

Statistical energy analysis (SEA) is a method where equations of energy equilibrium between member elements are statistically derived and solved. One feature of SEA is that it is unable to analyze each vibration mode of the structure separately, or produce a solution for specific points because it deals with energy flow as a whole for each member element. Since it is necessary to obtain a solution for each vibration mode of the structure for any given point, to obtain accurate prediction of structureborne noise, these specificities rule out the possibility of using this method for structure-borne noise prediction.

*) For example, for a noise of $1 \mathrm{kHz}$ the size of element should be less than $5.6 \mathrm{~cm}$.

**) This error is due to the computer's digital data being unable to express values below the significant range.

\section{Plan for developing of vibration analysis method}

Considering the aforementioned investigation results, any vibration analysis to be used in a method for predicting structure-borne should include the following properties:

(1) It should be capable of accurate structure vibration analysis in a $\mathrm{kHz}$ frequency range.
(2) It should be capable of analysis per vibration mode and be able to produce a solution for any specific location. Flexural vibration analysis is expected to satisfy these conditions for the following reasons.

(a) This method doesn't require vast degrees of equation even for structures composed of a large number of parts, because it uses a trial function which is defined considering the vibration mode of each member, which means that a numeric matrix with equation of motion of the structure in question can be obtained by treating each vibration mode as an element rather than dividing the spatial area into each fine element.

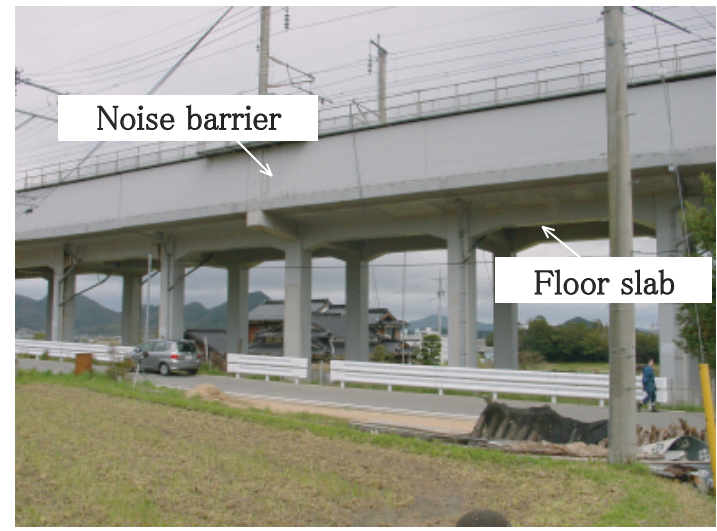

(a) Side view

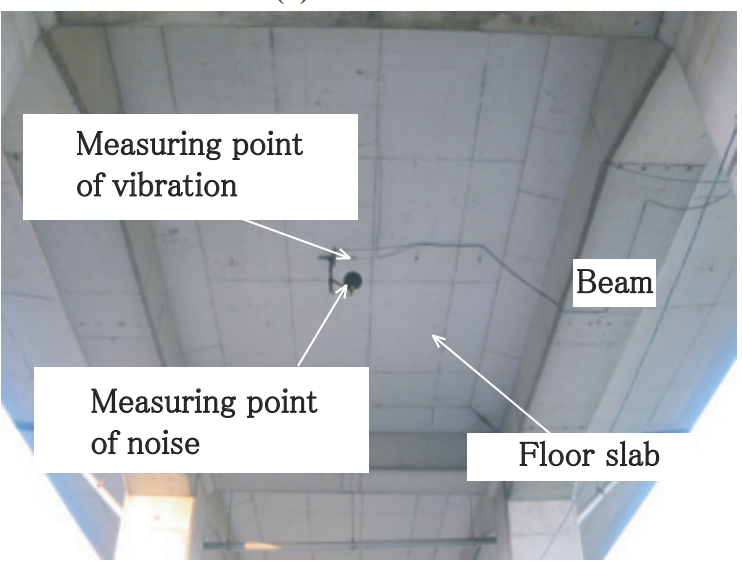

(b) Bottom view

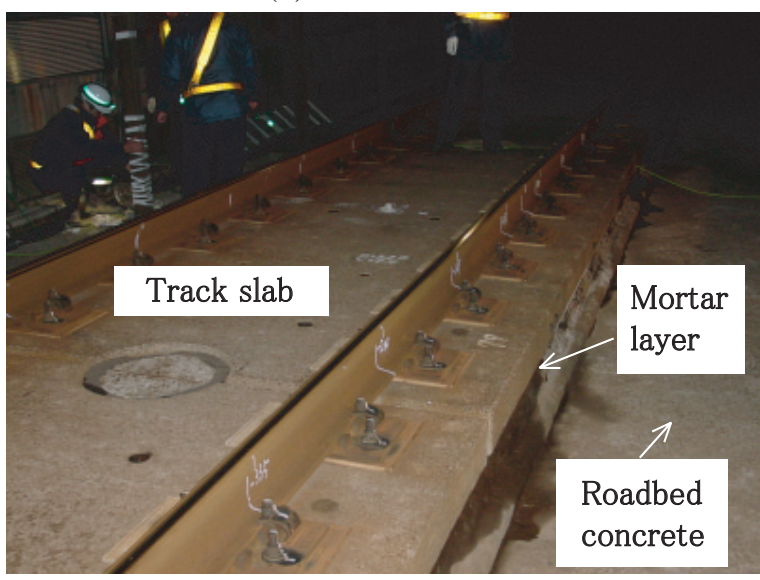

(c) Track view

Fig. 11 View of the subject concrete viaduct 
(b) Because the trial functions take into account vibration modes of the structure, the vibration mode's characteristics of the structure are roughly reflected at an early stage in the analysis.

(c) This method can analyze each vibration mode individually. Furthermore, it expresses displacement as a coordinate value which means that it is possible to pinpoint any specific location.

So far, this method has been applied to vibration analyses of individual structure members, and has been confirmed as having a tolerable level of accuracy. The next part of this paper investigates the applicability of the method to the prediction of structure-borne noise if it is extended to the whole of the structure.

\section{Flexural vibration analysis extended to the whole of the structure}

\subsection{Subject structure}

Details of the developed vibration analysis method are described in reference [4] (The plan is to have it published in English later). This paper, in the mean time, outlines the method and its underlying logic. Figure 1 is a picture of the concrete viaduct selected to be the analytical and experimental subject. It is a concrete viaduct constructed on a Shinkansen line and comprises slab track to which rails are fixed directly. The track has standard type track pads with a nominal spring constant of $60 \mathrm{MN} / \mathrm{m}$, no other forms of vibration damping, such as slab mats, were used.

\subsection{Modal analysis}

\subsubsection{Outline of the method for modal analysis}

Figure 2 illustrates the model used for analyzing con- crete viaduct vibration. For calculation efficiency, the structure is divided into analytical regions which are then modeled individually. The main processes involved in this method are described below:

(a) The major members comprising the structure are modeled into simple representations. For example, rails are represented by elastic beams and floor slabs and noise barriers by plates. The rail supporting layer in particular, usually comprising track pads, is represented by a continuous viscoelastic beam whose weight and rigidity in the $\mathrm{z}$ direction per unit length in rail direction are equal to that of an actual track pad. The reasons for representing what is actually a discrete rail support system by a continuous model are as follows: The load distribution effect of the pads means that from a physical point of view, it is as if the rail had continuous support. Furthermore, a continuous analytical model connects more logically to other models of members, such as track slabs, than would a discrete model

(b) Define the trial function which takes the form of displacement solution for each member model.

(c) Link the trial functions according to bonding conditions between adjacent members and boundary conditions.

(d) Derive the expressions, which are given as functions of the displacement, representing the energy of each modeled member.

(e) Apply the expressions obtained in (d) to the energy conservation law for the whole structure and then introduce the trial functions obtained in (b) into these equations. This makes it possible to derive the matrix form equation of motion.

(f) Solve the equation of motion to obtain the natural frequency and natural vibration mode which are the modal analysis solution.

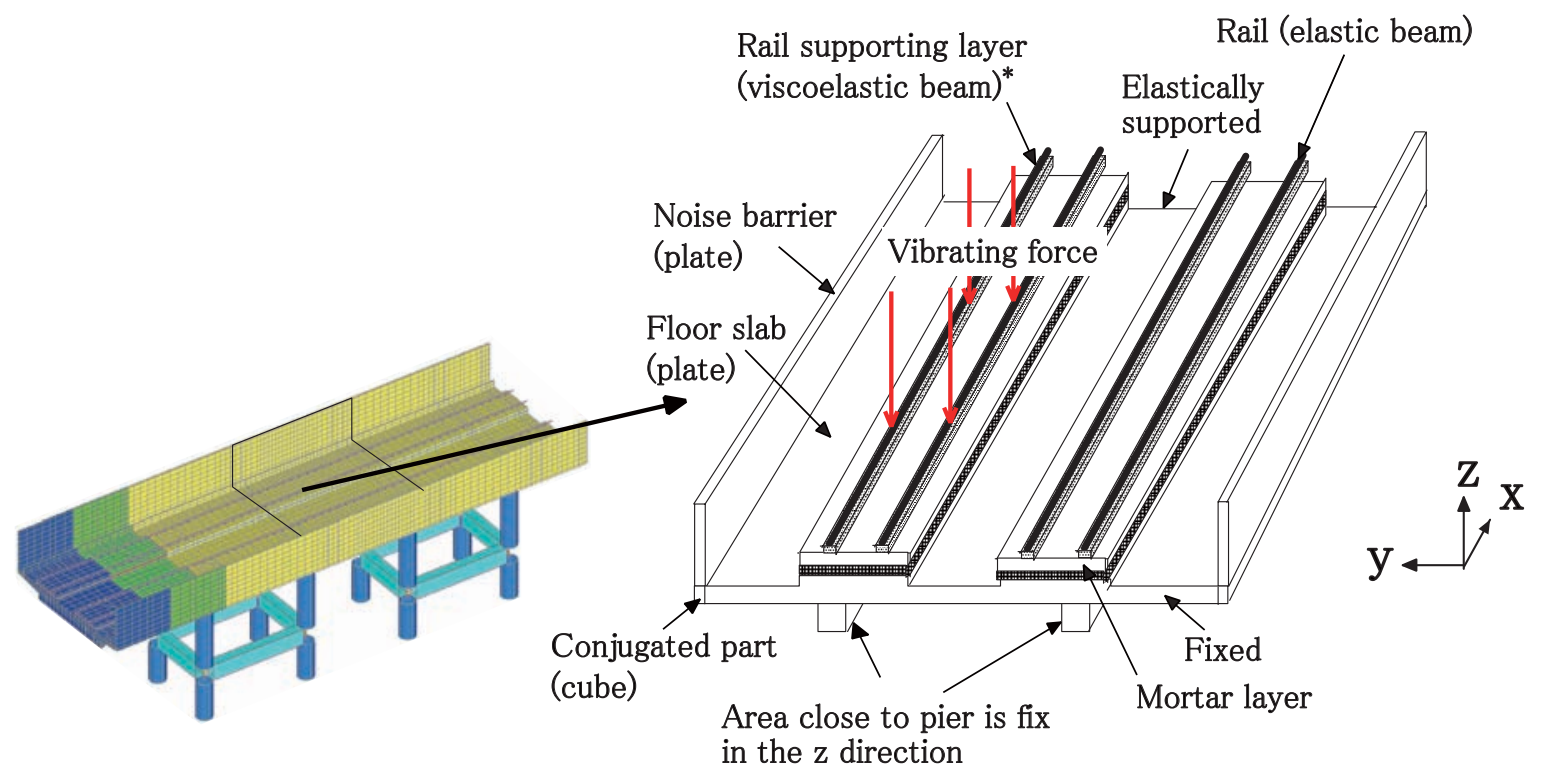

(a) FEM

(b) Developed method

Fig. 2 Model for vibration analysis of concrete viaduct 


\subsubsection{Definition of the trial function}

This section examines the expression for displacement, using trial functions. Let us express displacement for a given model $i$ in the $x$-direction as $u^{(i)}$, displacement in the $i$-direction as $v^{(i)}$, and in the $z$-direction as $w^{(i)}$. Hereafter in this paper, only expressions for displacement $\mathrm{w}$ are shown in full, and in the case of the other two types of displacement, only those parts which differ from $w$ are shown because the expressions of three displacements have almost similar formation. Moreover, even though this method covers one-dimensional (beam), two-dimensional (plate), and three-dimensional (cube) elements, almost all the examples given in this paper are threedimensional expressions.

Displacement is given as a product of variable separation as shown in (1).

$$
w^{(i)}(x, y, z, t)=\bar{w}^{(i)}(x, y, z) e^{j \omega t}
$$

(2).

$\bar{w}^{(i)}$ is expressed by a series of functions as shown in

$$
\bar{w}^{(i)}(x, y, z)=\sum_{k=1}^{N_{k}^{(i)}} \sum_{\ell=1}^{N_{\ell}^{(i)}} \sum_{m=1}^{N_{m}^{(i)}} W_{k \ell m}^{(i)} X_{k}^{(i)}(x) Y_{\ell}^{(i)}(y) Z_{m}^{(i)}(z)
$$

where $X_{k}^{(i)}(x), Y_{\ell}^{(i)}(y), Z_{m}^{(i)}(z)$ are the base functions, $W_{k \ell m}^{(i)}$ is the undetermined coefficient (determined by solving the equation of motion later), $N_{k}^{(i)}, N_{\ell}^{(i)}, N_{m}^{(i)}$ is the number needed for calculation (a standard number between $30 \sim 50$ is required for a calculation range $63 \mathrm{~Hz} \sim 2 \mathrm{kHz}), \bar{u}^{(i)}, \bar{v}^{(i)}$ are obtainable by modifying the undetermined coefficient to $U_{k \ell m}^{(i)}, V_{k \ell m}^{(i)}$ respectively.

Base functions are obtained by considering the vibration modes of each member of the structure estimated from the characteristics and shape values of each member. In the case of this study's cube model, the base function is defined from the eigenfunction of Euler beams, cut out from the members' cross sections along the individual $x, y, z$ axes. The vibration mode characteristics for each axial direction of the cubic isotropic member are roughly reflected in this function.

The base functions $X_{m}^{(i)}(x), Y_{m}^{(i)}(y), Z_{m}^{(i)}(z)$ are given by the following equations:

$$
\begin{aligned}
X_{k}^{(i)}(x)= & B_{k x 1}^{(i)} \cos \left(\theta_{k x}^{(i)} x\right)+B_{k x 2}^{(i)} \sin \left(\theta_{k x}^{(i)} x\right) \\
& +B_{k x 3}^{(i)} \cosh \left(\theta_{k x}^{(i)} x\right)+B_{k x 4}^{(i)} \sinh \left(\theta_{k x}^{(i)} x\right) \\
Y_{\ell}^{(i)}(y)= & B_{\ell y 1}^{(i)} \cos \left(\theta_{\ell y}^{(i)} y\right)+B_{\ell y 2}^{(i)} \sin \left(\theta_{\ell y}^{(i)} y\right) \\
& +B_{\ell y 3}^{(i)} \cosh \left(\theta_{\ell y}^{(i)} y\right)+B_{\ell y 4}^{(i)} \sinh \left(\theta_{\ell y}^{(i)} y\right) \\
Z_{m}^{(i)}(z)= & B_{m z 1}^{(i)} \cos \left(\theta_{m z}^{(i)} z\right)+B_{m z 2}^{(i)} \sin \left(\theta_{m z}^{(i)} z\right) \\
& +B_{m z 3}^{(i)} \cosh \left(\theta_{m z}^{(i)} z\right)+B_{m z 4}^{(i)} \sinh \left(\theta_{m z}^{(i)} z\right)
\end{aligned}
$$

where $B_{k x 1}^{(i)} \sim B_{k x 4}^{(i)}, B_{\ell y 1}^{(i)} \sim B_{\ell y 4}^{(i)}, B_{m z 1}^{(i)} \sim B_{m z 4}^{(i)}$ are coefficients, the relationships among which are so determined as to satisfy the bonding and boundary conditions in the directions of $x, y, z$ individually, and $\theta_{k x}^{(i)}, \theta_{\ell y}^{(i)}, \theta_{m z}^{(i)}$ are the characteristic solutions of the Euler beam equations, which are determined by the material properties such as young's modulus, size values, and so on. The components of the aforementioned displacement are then arranged and expressed in vector form. Vector transformation of displacement in the case of the three-dimensional model is expressed by the following equation.

$$
\begin{aligned}
& \left\{u v w^{(i)}\right\}=\left(\bar{u}^{(i)}, \bar{v}^{(i)}, \bar{w}^{(i)}\right)^{T} e^{j \omega t}=\left[\phi_{k \ell m}^{(i)}\right]\left\{U V W^{(i)}\right\} e^{j \omega t} \\
& {\left[\phi_{k \ell m}^{(i)}\right]=\left[\begin{array}{ccc}
<\phi_{k \ell m}^{(i)}> & <0> & <0> \\
<0> & <\phi_{k \ell m}^{(i)}> & <0> \\
<0> & <0> & <\phi_{k \ell m}^{(i)}>
\end{array}\right]} \\
& <\phi_{k \ell m}^{(i)}>=<X_{k}^{(i)}(x) Y_{\ell}^{(i)}(y) Z_{m}^{(i)}(z)> \\
& \left\{U V W^{(i)}\right\}=\left(U_{111}^{(i)}, \cdots, U_{k \ell m}^{(i)}, \cdots, U_{N_{k}^{(i)} N_{\ell}^{(i)} N_{m}^{(i)}}^{(i)}, \cdots, V_{k \ell m}^{(i)}, \cdots\right. \\
& , V_{N_{k}^{(i)} N_{\ell}^{(i)} N_{m}^{(i)}}^{(i)}, \cdots, W_{k \ell m}^{(i)}, \cdots, W_{N_{k}^{(i)} N_{\ell}^{(i)} N_{m}^{(i)}}^{(T)}
\end{aligned}
$$

where \{\} denotes the column vector, and $<>$ denotes the row vector.

\subsubsection{Derivation of the equation of motion and solution}

Next, the equation of motion is derived from the energy conservation law. This study considers that vibration damping values of actual systems are so small that they do not have any significant influence the vibration mode of the system and are therefore ignored for the purposes of this modal analysis. The principal of the energy conservation law is given in the following equation:

$$
\begin{aligned}
& \int_{t_{0}}^{t_{1}} \delta(T-U) d t=0 \\
& T=\sum_{i}^{n} T^{(i)}, U=\sum_{i}^{n} U^{(i)}
\end{aligned}
$$

where $T$ is the kinetic energy of whole structure (J), $T^{(i)}$ is the kinetic energy of model $i(\mathrm{~J}), U$ is the strain energy of whole structure $(J), U^{(i)}$ is the strain energy of model $i$ $(\mathrm{J}), n$ is the number of models in the whole of structure, and $t_{0}, t_{1}$ are the supposed times when the principal of energy conservation is applied (s).

The expressions for kinetic and strain energy in each model comprise the displacement of $u, v, w$, and are applied to (5) and (6). Moreover, if the trial functions, one instance of which is given by (4), are substituted into the composed equation, and the integral is executed, then the equation of motion in matrix form is obtained as shown in the following equation:

$$
\sum_{i=1}^{n}\left(-\omega^{2}\left[M^{(i)}\right]+\left[K^{(i)}\right]\right)\{U V W\}=0
$$

where $\left[M^{(i)}\right]$ is the mass matrix of model $i,\left[K^{(i)}\right]$ is the stiffness matrix of model $i$, and $\{U V W\}$ is the vector obtained by conjugating vector $\left\{U V W^{(i)}\right\}$ given by (4) in all models of the overall structure.

The natural frequencies and vibration modes are obtained by solving (7). During this process, using the substructure synthesis method shown in reference [5] as a basis, makes it possible to obtain an effective means to calculate vibrations for the whole structure. This approach means that all the degrees of freedom of the whole structure do not have to be handled together; rather a part of degrees of freedom in equation of motion of some members are canceled to allow the calculation with fewer degrees of freedom*. As the result, the total number of degrees in equation of motion of the whole structure decreases, and the effectiveness of the calculation is promoted.

As demonstrated in (4) and (7), the equation of mo- 
tion in matrix form is not given by dividing the spatial area of the analytical subject in to fine elements; it is a matrix given by the degrees of equation depending on the number of vibration modes. If this method only considers the vibration modes required for the structure vibration analysis in the frequency band to be analyzed $(63 \mathrm{~Hz}$ $\sim 2 \mathrm{kHz}$ ), there are far fewer degrees of equation than would be needed for FEM where the structure would have to be divided to fine elements to analyze the vibration for higher frequencies up to $2 \mathrm{kHz}$.

Moreover, it is possible to obtain the displacement value for any vibration mode of member in a structure at any specific location because the displacement solution is expressed in the formula (2).

*) Based on the equation of dynamic equilibrium, some degrees of freedom of displacement are expressed by the functions representing the relationship between them and other degrees of freedom of displacement which are dynamically related to them and are canceled by these functions. The canceled degrees of freedom of displacement are expressed again by the above mentioned function after obtaining the solution.

\subsection{Forced vibration analysis}

After the modal analysis performed above, the solution of forced vibration displacement is sought by applying the vibrational force to the modal analysis solution and the solution of forced vibration velocity is sought by differentiating the above solution once with time. In the case of the forced vibration solution, vibration damping through the rail supporting layer, mortar and concrete layers are considered. Damping values for the rail supporting layer and mortar were determined through resonance experiments, and the value for the concrete layer was quoted from references [6].

In this analysis, the vibrational force was supposed to be applied in constant locations, shown in Fig. 2. The developed method is regarded as a type of frequency response analysis and as such it is common practice to exert the vibrational force in a constant location because the analysis aims to analyze the stationary and maximum state of vibration. The locations in question correspond to the wheels of the vehicle at the moment when the center of bogie of the vehicle passes over the center of floor slab on the structure be studied. The amplitude of vibrating force was determined by the contact force between wheel and rail which is estimated on the basis of a contact filter model [7] (that relates the roughness of wheel and rail, rail vibration and the contact force based on a physical model of wheel/rail system). All four vibrating force phases were set the same.

\subsection{Verification of the developed method for vibration analysis}

To verify the accuracy of the developed method for vibration analysis described above, the vibration velocity of the viaduct being studied was calculated for the center point on the underside of a floor slab of the viaduct and compared with actual measured values. In addition to the developed method, the values calculated by FEM are also shown in Fig. 3. The analysis frequency range was $63 \mathrm{~Hz}$ $\sim 2 \mathrm{kHz}$ according to the presumed value of contact force. In addition, a corrective value $\mathrm{A}$ was applied to the vibration velocity to ensure that its frequency weighting characteristics were consistent with those of the noise mentioned later, since vibration velocity correlates with noise in general.

The outline of the FEM analysis is as follows: the analytical model is shown in Fig.2 (a), where the rail and pier are modeled by beam elements and track slab, floor slab and noise barrier are modeled with solid elements. The minimum size of the elements was about $0.3 \mathrm{~m}$, and the total number of nodes was 22,636 . The analytical approach applied was NX Nastran version 4.0.

Figure 3 demonstrates that the calculated values obtained with the developed method remained stable within $\pm 5 \mathrm{~dB}$ compared with the measured values which varied from $63 \mathrm{~Hz}$ to $2 \mathrm{kHz}$. On the other hand, the calculated values by FEM fluctuate more as the frequency exceeds $250 \mathrm{~Hz}$, and deviations from measured values is greater than $5 \mathrm{~dB}$ in most frequency bands over $250 \mathrm{~Hz}$.

These results confirm that the developed method for vibration analysis has a tolerable accuracy up to $2 \mathrm{kHz}$, and can be applied in predicting structure-borne noise.

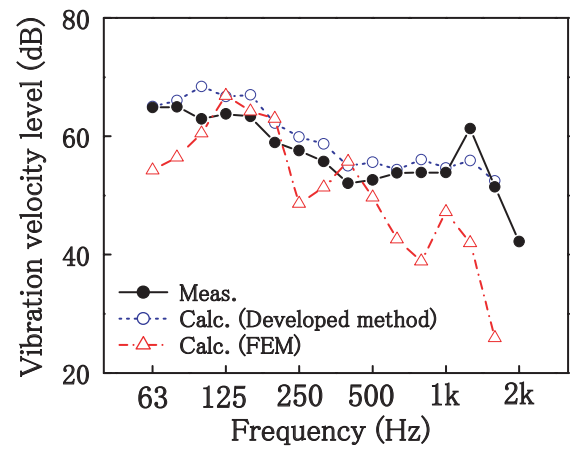

\section{Fig. 3 Calculating and measuring results of vibration of concrete viaduct \\ - The center point on the underside of a floor slab -}

\section{Prediction of structure-borne noise}

\subsection{The outline of the method for prediction of noise}

In the viaduct being studied, most of the members related to structure-borne noise are plate elements. Preliminary examinations and other previous studies on the other hand indicate that pier vibrations are small, and the subsequent contribution to structure-borne noise is considered to be negligible. Considering these factors, this analysis examines how to calculate the noise radiated from vibrating plates such as floor slabs and noise barriers. In this analysis, beams embedded in the floor slabs are regarded as a part of the floor slab.

The noise radiated from vibrating plates is considered to be a sum of noise radiated from infinitesimal semispherical oscillators which are derived by dividing the plate into infinitesimal areas as shown in Fig. 4 [8]. Then, $d P_{w}(\mathrm{~Pa})$, the sound pressure radiated from the infinitesimal oscillator, is given by the following equation. 


$$
d P_{w}=\frac{j \rho_{a} c_{a} k_{a}}{2 \pi r} u_{w} e^{j\left(\omega t-k_{a} r^{\prime}\right)} d S
$$

where $u_{w}$ is the vibration velocity of vibrating plate $(\mathrm{m} / \mathrm{s}), \rho_{a}$ is the density of air $\left(\mathrm{kg} / \mathrm{m}^{3}\right), c_{a}$ is the velocity of the noise $(\mathrm{m} / \mathrm{s}), k_{a}$ is the wave number of air $(1 / \mathrm{m}), r$ is the distance between the center of the oscillator and the sound pressure calculation point $(\mathrm{m}), \rho_{a} c_{a}$ is generally called the acoustic impedance.

If the solution of the developed method for forced vibration is applied to $u_{w}$ in (8), then $d P_{w}$ is calculated. If $d P_{w}$ is integrated over the vibrating plate area, then $P_{w}(\mathrm{~Pa})$, the sound pressure radiated from the vibrating plate, is obtained as follows:

$$
P_{w}=\int_{0}^{a} \int_{0}^{b} \frac{j \rho_{a} c_{a} k_{a}}{2 \pi r} u_{w} e^{j\left(\omega t-k_{a} r^{r}\right)} d x d y
$$

If $P_{w}$ is integrated over a closed space* area around the vibrating plate, then the power of structure-borne noise radiated from this plate is obtained. Moreover, the power of structure-borne noise of the whole of structure is obtained from the sum of all powers of noise radiated from each plate being studied, such as floor slabs and noise barriers.

*) Generally, the space is set as a sphere whose center is the center of analytical coordinates and diameter is the distance between the center of analytical coordinates and the sound pressure calculation point $(r$ shown in Fig. 4).

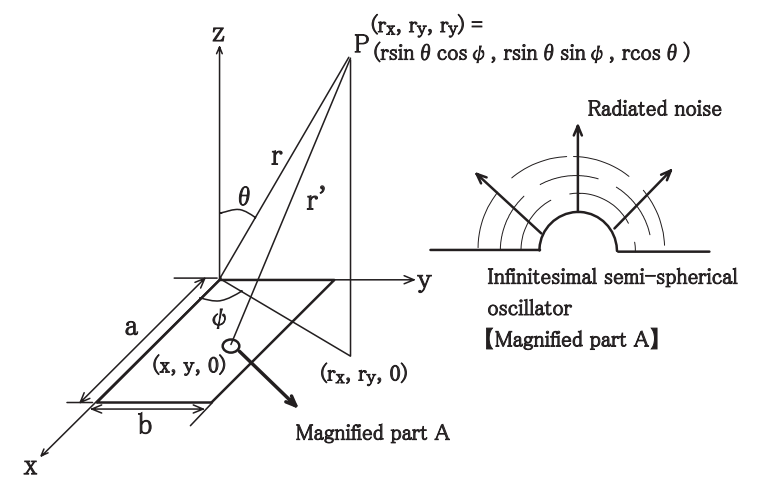

Fig. 4 Analytical model for prediction of noise

\subsection{Verification of proposed method for prediction of noise}

To verify the accuracy of the abovementioned noise prediction method noise in the vicinity of the center point on the underside of the floor slab of the viaduct being studied was calculated (only $300 \mathrm{~mm}$ beneath the center point of the floor slab) and compared with the measured value. Figure 5 illustrates the results. Figure 5 shows that the calculated values obtained with the proposed method remained within $\pm 5 \mathrm{~dB}$ compared with the measured values in the range $63 \mathrm{~Hz}$ to $2 \mathrm{kHz}$, confirming that the proposed method has a tolerable level of accuracy up to $2 \mathrm{kHz}$. On the other hand, in some frequency bands, the differences between the calculated and measured values amount to almost $5 \mathrm{~dB}$, which indicates the need to improve the accuracy of the noise prediction method. One method to improve accuracy in noise prediction of noise

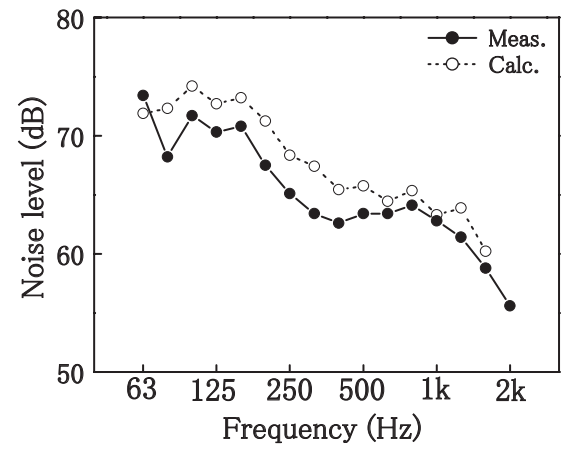

\section{Fig. 5 Calculating and measuring results of noise near the concrete viaduct}

- at a distance of $300 \mathrm{~mm}$ from the center point on the underside of the floor slab -

could be to improve the method used for estimating vibrational force, by trying to apply models other than the contact filter model.

\section{Conclusion}

In this study of the concrete viaduct, on which trackside noise needs to be reduced, various structure vibration analyses and structure-borne noise prediction methods were examined. This led to the following conclusions:

(1) A new vibration analysis method, which is a flexural vibration analysis extended to a whole structure was developed. It has the following characteristics: the structure is divided to main members and each member is represented by a simple model to calculate the vibration of structure efficiently. The matrix-form equation of motion is derived without spatially dividing the structure, by introducing a trial function for each member model which considers each member's vibration mode.

(2) As the result of comparison of the measured and calculated values of the center of the underside of the floor slab of a concrete viaduct constructed on a Shinkansen line, the developed method for vibration analysis was confirmed as having a tolerable level of accuracy up to $2 \mathrm{kHz}$.

(3) A new method for the prediction of structure-borne noise using the solutions from the aforementioned method of vibration analysis was proposed. The proposed method calculates the noise radiated from vibrating plate by multiplying the solutions from the developed method of vibration analysis with acoustic impedance.

(4) As the result of comparison of the measured and calculated values of noise in the vicinity of the center point on the underside of the floor slab of the viaduct being studied, it demonstrated that the proposed method can be used as a practical method to predict structure-borne noise. On the other hand, it is also recognized that the accuracy of the noise prediction method needs to be improved for more precise prediction. Plans are in place to examine how to achieve such improvement in a method for predicting struc- 
ture-borne noise.

\section{References}

[1] HANSAKA,M. et al., "The analysis of bending vibration of plate with magnetic rubber damper with a constraining layer," RTRI Report, Vol. 16, No.12, pp. 2328, 2002 (in Japanese).

[2] JANSSENS, M.H.A. and THOMPSON, D.J., "A Calculation model for the noise from steel railway bridges," Journal of Sound and Vibration, Vol.193, No.1, pp.295-305, 1996.

[3] ODA, M. et al., "Noise prediction and reduction for an open-floored steel railway bridge," KAWASAKIJYUKO-KIHOU, No.139, pp.43-48, 1998 (in Japanese).
[4] HANSAKA, M., et al., "Vibration and noise analysis of railway concrete viaduct," Dynamic \& Design conference by JSME, No.631 (CD-ROM), 2008 (in Japanese).

[5] NAGAMATSU, A. and OOKUMA, M., Substructure synthesis method, BAIFUKAN, p.354, 1991 (in Japanese).

[6] MATSUHISA, H., "Damping material for machine," Journal of INCE, Vol.23, No.6, pp.391-394, 1999 (in Japanese).

[7] THOMPSON, D. J., and JONES, C.J.C., "A review of the modeling of wheel/rail noise generation," Journal of Sound and Vibration, Vol.231, No.3, pp.519-536, 2000.

[8] WALLACE, C.E., "Radiation Resistance of a Baffled Beam," Journal of Acoustic Society of America, Vol.51, No.3, pp.936-945, 1972. 